\title{
FLAT REGULAR QUOTIENT RINGS
}

\author{
BY \\ VASILY C. CATEFORIS
}

0 . Introduction and notation. In this paper we study the condition that the maximal right quotient (MRQ) ring $Q[10, \mathrm{p} .106]$ of a right nonsingular ring $R$ with 1 is flat as a left $R$-module. It is known [11, p. 134] that if $Q$ is the classical right quotient ring of $R$, then $Q$ is flat as a left $R$-module. This is not always the case with the MRQ ring of $R$ : in $\$ 2$ we obtain an ideal theoretic characterization (Theorem 2.1) and a module theoretic characterization (Theorem 2.2) of a right nonsingular ring $R$, all of whose regular right quotient rings are flat as left $R$-modules; we also indicate the existence of a class of commutative rings $R$, whose singular ideal is zero and for which the maximal quotient ring is not $R$-flat.

Throughout this paper $R$ denotes an associative ring with identity. A right $R$-module $M$ is denoted $M_{R}$; all $R$-modules are unitary.

Let $N_{R}$ and $M_{R}$ be modules such that $N_{R} \subseteq M_{R}$. We say that $N_{R}$ is large in $M_{R}$ ( $M_{R}$ is an essential extension of $N_{R}$ ) if $N_{R}$ intersects nontrivially every nonzero submodule of $M_{R}$. A right ideal $I$ or $R$ is large in $R$ if $I_{R}$ is large in $R_{R}$.

For any module $M_{R}, L\left(M_{R}\right)$ denotes the lattice of large submodules of $M_{R}$.

Let $M_{R}$ be a module. We denote by $Z\left(M_{R}\right)$ the singular submodule of $M_{R}$. If for any $x \in M$ we set $(0: x)=\{r \in R \mid x r=0\}$, then

$$
Z\left(M_{R}\right)=\left\{x \in M \mid(0: x) \in L\left(R_{R}\right)\right\} .
$$

In particular $Z\left(R_{R}\right)$ denotes the singular (two-sided) ideal of $R_{R}$ and $R$ is right nonsingular if $Z\left(R_{R}\right)=(0)$.

A ring $S$ containing $R$ is a right quotient ring of $R$, if $R_{R} \in L\left(S_{R}\right)$.

Let $S$ be a right quotient ring of $R$. It is easy to see that if $I_{R} \in L\left(R_{R}\right)$, then $I S \in L\left(S_{S}\right)$ and if $A_{S} \in L\left(S_{S}\right)$, then $(A \cap R)_{R} \in L\left(R_{R}\right)$. For a module $M_{R}, M \otimes_{R} S$ is a right $R$ - and $S$-module; it follows easily from the preceding observation that $Z\left(\left(M \otimes_{R} S\right)_{R}\right)=Z\left(\left(M \otimes_{R} S\right)_{S}\right)$ and hereafter we write $Z(M i \otimes S)$. Also for any left $R$-module ${ }_{R} N$ we write $M \otimes N$ for $M \otimes_{R} N$ if no ambiguity arises.

A ring is regular in the sense of Von Neumann [16]. Characterizations of flatness used frequently in this paper can be found in [10, pp. 132-135]. For all homological notions the reader may consult [2].

This paper constitutes a portion of the author's doctoral dissertation at the University of Wisconsin. The author is deeply indebted to Professor F. L. Sandomierski, his advisor, for supplying many of the ideas included in what follows.

Received by the editors March 13, 1968 and, in revised form, May 27, 1968. 
1. Preliminaries. Let $R$ be a ring. In the following proposition we record some well-known facts about large submodules. The proof of all but part (v) can be essentially found in [7], [8], [13]. We prove part (v) below.

Proposition 1.1. Let $M$ and $N$ be right $R$-modules. The following statements are then true:

(i) If $A_{R}, B_{R} \in L\left(M_{R}\right)$, then $A \cap B, A+B \in L\left(M_{R}\right)$.

(ii) If $A_{R} \in L\left(M_{R}\right)$ and $B_{R} \subseteq M_{R}$ such that $A_{R} \subseteq B_{R}$, then $B_{R} \in L\left(M_{R}\right)$.

(iii) If $A_{R} \subseteq M_{R}$, then there exists $B_{R} \subseteq M_{R}$ such that $A \cap B=(0)$ and $A+B$ is large in $M_{R}$.

(iv) If $f \in \operatorname{Hom}_{R}\left(M_{R}, N_{R}\right)$ and $A_{R} \in L\left(N_{R}\right)$, then $f^{-1}\left(A_{R}\right) \in L\left(M_{R}\right)$.

(v) If $f \in \operatorname{Hom}_{R}\left(M_{R}, N_{R}\right)$, $f$ an epimorphism, $Z\left(N_{R}\right)=(0)$ and $A_{R} \in L\left(M_{R}\right)$, then $f\left(A_{R}\right) \in L\left(N_{R}\right)$.

(vi) If $A_{1}, \ldots, A_{n} \in L\left(M_{R}\right)$ and $x_{1}, \ldots, x_{n} \in M_{R}$ then

$$
I=\left\{r \in R \mid x_{i} r \in A_{i}, i=1, \ldots, n\right\} \in L\left(R_{R}\right) .
$$

Proof. (v). Let $0 \neq n \in N$ and pick $m \in f^{-1}(n)$. The right ideal $I_{R}=\{r \in R \mid m r \in A\}$ is large in $R$ by (vi), so that $n I_{R} \neq(0)$ as $Z\left(N_{R}\right)=(0)$. There exists, hence, $r \in I_{R}$ such that $n r \neq 0$ and since $m r \in A$ we have $f(m r)=n r \in f(A)$. Q.E.D.

In [3, p. 459] Chase calls a right $R$-module $M$ finitely related if there exists an exact sequence of right $R$-modules $0 \rightarrow K_{R} \rightarrow F_{R} \rightarrow M_{R} \rightarrow 0$ with $F_{R}$ free and both $F_{R}$ and $K_{R}$ finitely generated. We generalize this concept below:

Definition 1.2. A right $R$-module $M$ is essentially finitely generated $\left(M_{R}\right.$ is EFG) if there exist finitely many elements $m_{1}, \ldots, m_{n}$ of $M_{R}$ such that $\sum m_{i} R$ $\in L\left(M_{R}\right)$.

Definition 1.3. A right $R$-module $M$ is essentially finitely related ( $M_{R}$ is EFR) if there exists an exact sequence $0 \rightarrow K_{R} \rightarrow F_{R} \rightarrow M_{R} \rightarrow 0$ with $F_{R}$ finitely generated free and $K_{R}$ EFG.

If $M_{R}$ is EFR then any exact sequence $0 \rightarrow K_{R} \rightarrow F_{R} \rightarrow M_{R} \rightarrow 0$ will have the property that $K_{R}$ is EFG whenever $F_{R}$ is finitely generated free; this follows from a result of Schanuel's contained in [15, p. 369].

To obtain our main results (\$2) we need some lemmas:

LEMMA 1.4. Let $0 \rightarrow K_{R} \stackrel{i}{\rightarrow} M_{R} \stackrel{f}{\rightarrow} N_{R} \rightarrow 0$ be an exact sequence of right $R$-modules where $K=\operatorname{ker} f$ and $i$ is the inclusion map. We have:

(a) If $K_{R}, N_{R}$ are $E F G$ and $Z\left(M_{R}\right)=(0)$, then $M_{R}$ is $E F G$.

(b) If $Z\left(N_{R}\right)=(0)$ and $M_{R}$ is $E F G$, then $N_{R}$ is $E F G$.

Proof. (b) follows easily from Proposition 1.1(v), so we prove (a):

Let $\left\{k_{i}: i=1, \ldots, n\right\} \subset K_{R},\left\{n_{j}: j=1, \ldots, l\right\} \subseteq N_{R}$ (finite sets), such that $\sum k_{i} R \in$ $L\left(K_{R}\right)$ and $\sum n_{j} R \in L\left(N_{R}\right)$. Choose $m_{j} \in f^{-1}\left(n_{j}\right)$ for $j=1, \ldots, l$ and set $M_{R}^{\prime}=\sum_{i} k_{i} R$ $+\sum_{j} m_{j} R$. We show next that $M_{R}^{\prime} \in L\left(M_{R}\right)$. Let $0 \neq m \in M$. If $f(m)=0$ then $m \in K$ and there exists $r \in R$ such that $0 \neq m r \in \sum k_{i} R \subset M^{\prime}$. Assume, next, $f(m) \neq 0$. There exists $r \in R$ such that $0 \neq f(m) r=n_{1} r_{1}+\cdots+n_{l} r_{l} \in \sum_{j} n_{j} R$. Set $m^{\prime}=m_{1} r_{1}+\cdots+$ 
$m_{l} r_{l}$; clearly $m^{\prime} \neq 0$ and $f\left(m r-m^{\prime}\right)=0$. If $m r-m^{\prime}=0$ then $0 \neq m r=m^{\prime} \in M^{\prime}$, so assume $m r-m^{\prime} \neq 0$. Since $m r-m^{\prime} \in K$ it follows by Proposition 1.1(vi) that $I=$ $\left\{x \in R \mid\left(m r-m^{\prime}\right) x \in \sum_{i} k_{i} R\right\} \in L\left(R_{R}\right)$ and since $Z\left(M_{R}\right)=(0)$, there exists $t \in I$ such that $(m r) t \neq 0$. Thus $\left(m r-m^{\prime}\right) t=k \in \sum_{i} k_{i} R$ and $0 \neq m(r t)=m^{\prime}+k \in M^{\prime}$. We have shown that for every $0 \neq m \in M, M^{\prime} \cap m R \neq(0)$, hence $M^{\prime} \in L\left(M_{R}\right)$. Q.E.D.

Lemma 1.5. Suppose $R$ has a right quotient ring $Q$ and $K_{R}$ is an $R$-module such that $Z\left(K_{R}\right)=(0)$. If $K \otimes_{R} Q$ is finitely generated as a right $Q$-module, then $K_{R}$ is $E F G$.

Proof. Let $\left\{k_{i} \otimes 1: i=1, \ldots, n, k_{i} \in K\right\}$ be a set of generators for $K \otimes Q$. Let $L$ be the submodule of $K$ generated by the elements $k_{1}, \ldots, k_{n}$. We show next that $L_{R}$ is large in $K_{R}$. Since $Z\left(K_{R}\right)=(0)$, the sequence $(0) \rightarrow K \rightarrow K \otimes Q$ is exact [13, Proposition 2.2], where the mapping is the canonical one: $k \rightarrow k \otimes 1$. Now let $0 \neq k \in K$; we have $k \otimes 1=\sum_{i=1}\left(k_{i} \otimes 1\right) q_{i}$. The right ideal $I_{R}=\left\{r \in R \mid q_{i} r \in R, i=\right.$ $1, \ldots, n\}$ is large in $R$ by Proposition 1.1 (vi) and since $k \otimes 1$ is a nonsingular element of $K \otimes Q$ (being the image of one under monomorphism) it follows that $(k \otimes 1) I_{R} \neq(0)$. Hence there is $r \in I_{R}$ such that $0 \neq(k r) \otimes 1=(k \otimes 1) r=\sum_{i} k_{i} \otimes$ $q_{i} r=\sum_{i} k_{i}\left(q_{i} r\right) \otimes 1$. It follows that $0 \neq k r=\sum k_{i}\left(q_{i} r\right) \in L$ and thus $L_{R}$ is large in $K_{R}$. Q.E.D.

The following lemma due to Kaplansky [9, Lemma 4, p. 376] is stated here because of its frequent use in the sequel:

LEMMA 1.6 (KAPLANSKY). If $P$ is a projective right (left) module over a regular ring $R$, then any finitely generated submodule of $P$ is a direct summand of $P$.

We shall need the following characterization of a module $M_{R}$ which is EFR.

Proposition 1.7. Let $R$ be a ring with $Z\left(R_{R}\right)=(0)$ and a right quotient ring $Q$ which is regular. A finitely generated right $R$-module $M$ is EFR if and only if $M \otimes Q$ is projective as a right $Q$-module.

Proof. Only if. Let $0 \rightarrow K_{R} \stackrel{i}{\rightarrow} F_{R} \stackrel{f}{\rightarrow} M_{R} \rightarrow 0$ be an exact sequence with $F_{R}$ finitely generated free and $K_{R}$ EFG. Let $k_{1}, \ldots, k_{n}$ be finitely many elements of $K$ such that $K^{\prime}=\sum_{i=1}^{n} k_{i} R \in L\left(K_{R}\right)$. It suffices to show that the exact sequence $K \otimes Q \stackrel{i^{*}}{\rightarrow} F \otimes Q \stackrel{\stackrel{*}{*}^{\prime}}{\rightarrow} M \otimes Q \rightarrow 0$ splits or equivalently $i^{*}(K \otimes Q)$ is a direct summand of $F \otimes Q$. Let $A$ be the right $Q$-submodule of $F \otimes Q$ generated by $k_{i} \otimes 1, i=1, \ldots, n$ in $F \otimes Q$. By Lemma $1.6 A_{Q}$ is a direct summand of $F \otimes Q$. The proof of this part will be complete if we show that $A_{Q}$ is large in $i^{*}(K \otimes Q)$. Thus let $0 \neq \sum_{i} x_{i} \otimes q_{i} \in i^{*}(K \otimes Q), \quad x_{i} \in K, \quad q_{i} \in Q$. By Proposition 1.1(vi), $I=\left\{r \in R \mid q_{i} r \in R\right.$, all $\left.i\right\} \in L\left(R_{R}\right)$ and since $Z\left(i^{*}(K \otimes Q)\right)=(0)$ as $Z(F \otimes Q)=(0)$, there exists $t \in I$ such that $0 \neq\left(\sum_{i} x_{i} \otimes q_{i}\right) t=\sum_{i} x_{i} \otimes\left(q_{i} t\right)=\sum x_{i}\left(q_{i} t\right) \otimes 1 \in A_{Q}$. It follows that $A_{Q}$ is large in $i^{*}(K \otimes Q)$.

If part. Using the notation above we see that since $M \otimes Q$ is right $Q$-projective, $i^{*}(K \otimes Q)$ is a direct summand of $F \otimes Q$ and hence it is finitely generated as a 
right $Q$-module. Let $\left\{x_{i} \otimes 1: x_{i} \in K, i=1, \ldots, n\right\}$ be a set of generators for $i^{*}\left(K \otimes_{R} Q\right)$. The module $K_{R}^{\prime}=\sum_{i} x_{i} R$ is, then, large in $K_{R}$. Indeed let $0 \neq k \in K$. As $K \subseteq F$ it is clear that $k \otimes 1 \neq 0$ in $F \otimes Q$ and hence $0 \neq k \otimes 1=\sum\left(x_{i} \otimes 1\right) q_{i}=$ $\sum_{i} x_{i} \otimes q_{i} \in i^{*}(K \otimes Q)$. As before $I=\left\{r \in R \mid q_{i} r \in R\right.$, all $\left.i\right\} \in L\left(R_{R}\right)$ so there exists $t \in I$ such that $0 \neq(k \otimes 1) t=\sum_{i} x_{i}\left(q_{i} t\right) \otimes 1$. In particular $\left[k t-\sum x_{i}\left(q_{i} t\right)\right] \otimes 1=0$ and hence $0 \neq k t=\sum_{i} x_{i}\left(q_{i} t\right) \in K_{R}^{\prime}$. We have $K_{R}^{\prime} \in L\left(K_{R}\right)$ and hence $K_{R}$ is EFG or $M_{R}$ is EFR. Q.E.D.

LEMMA 1.8. Let $R$ be a ring with $Z\left(R_{R}\right)=(0)$ and let $Q$ be a right quotient ring of $R$. If $I$ is any right ideal of $R$, then $Z(I \otimes Q)=\operatorname{ker}(I \otimes Q \rightarrow R \otimes Q)$.

Proof. If $A$ and $B$ are right $R$-modules and $f \in \operatorname{Hom}_{R}\left(A_{R}, B_{R}\right)$, then it is clear that $f\left(Z\left(A_{R}\right)\right) \subseteq Z\left(B_{R}\right)$. Now $R \otimes Q \cong Q$ and $Z\left(Q_{R}\right)=(0)$ as $Z\left(R_{R}\right)=(0)$, so $Z(I \otimes Q) \subseteq \operatorname{ker}(I \otimes Q \rightarrow R \otimes Q)$.

Conversely let $u=\sum x_{i} \otimes q_{i} \in I \otimes Q, x_{i} \in I, q_{i} \in Q$ such that $\sum x_{i} \otimes q_{i} \rightarrow \sum x_{i} q_{i}$ $=0$. For every $t \in J=\left\{r \in R \mid q_{i} r \in R\right.$, all $\left.i\right\}$ we have $\left(\sum x_{i} \otimes q_{i}\right) t=\sum x_{i}\left(q_{i} t\right) \otimes 1=0$. Since $J \in L\left(R_{R}\right)$ we have $(0: u) \in L\left(R_{R}\right)$, by Proposition 1.1, and hence $u \in$ $Z(I \otimes Q)$. Thus $\operatorname{ker}(I \otimes Q \rightarrow R \otimes Q) \subseteq Z(I \otimes Q)$ and this completes the proof of the lemma. Q.E.D.

We need the following characterization of flatness of a regular right quotient ring:

THEOREM 1.9. For any ring $R$ with $Z\left(R_{R}\right)=(0)$, the following statements are equivalent, for any regular right quotient ring $Q$ of $R$ :

(a) $Q$ is flat as a left $R$-module.

(b) $Z(I \otimes Q)=(0)$ for every finitely generated right ideal $I$.

(c) $I \otimes Q$ is $Q$-projective for every finitely generated right ideal of $R$.

Proof. (a) implies (b). By Lemma 1.8 and flatness of ${ }_{R} Q$.

(b) implies (c). For any finitely generated right ideal $I$ or $R$, the sequence $0 \rightarrow I \otimes Q \rightarrow R \otimes Q$ is exact by Lemma 1.8 and (b). Now $I \otimes Q$ is projective as a right $Q$-module follows from Lemma 1.6.

(c) implies (a). For any finitely generated right ideal $I$ of $R, I \otimes Q$ is a submodule of a $Q$-free module [2, p. 6], hence $Z(I \otimes Q)=(0)$ as $Z\left(Q_{Q}\right)=(0)$. Now (a) follows from Lemma 1.8. Q.E.D.

The proof of the following is essentially contained in [6, p. 953]; it is given here for completeness.

Lemma 1.10. If $M$ is a flat left $R$-module and $I, J$ are right ideals of $R$, then $(I \cap J) M=I M \cap J M$ (the right-left symmetric of this also holds).

Proof. The image of $(I \cap J) \otimes M$ in $(I+J) \otimes M$ is equal to the intersection of the images of $I \otimes M$ and $J \otimes M$ in $(I+J) \otimes M$, all images under homomorphisms induced by the obvious inclusions [1, Lemme 7, p. 32]. Since ${ }_{R} M$ is flat, the mapping $\sum a_{i} \otimes m_{i} \rightarrow \sum a_{i} m_{i}$ from $A \otimes M$ to $A M$, for any right ideal $A_{R}$ of $R$, 
is an isomorphism [10, p. 132] (of abelian groups); under such an isomorphism from $(I+J) \otimes M$ to $(I+J) M$ we obtain $(I \cap J) M=I M \cap J M$. Q.E.D.

The following is a generalization of a lemma by Chase [3, Lemma 2.2, p. 462]:

Lemma 1.11. Let $M$ be any right $R$-module, $N=\sum_{i=1}^{n} m_{i} R$ a finitely generated submodule of $M_{R}$ and $m \in M$. Set $N^{*}=N+m R$ and $F=x_{1} R \oplus \cdots \oplus x_{n} R \oplus x_{n+1} R$, a free right $R$-module. Let $f: F \rightarrow N^{*}$ be the $R$-epimorphism defined $f\left(x_{i}\right)=m_{i}$, $1 \leqq i \leqq n$ and $f\left(x_{n+1}\right)=m$. Set $K=\operatorname{ker} f, F^{\prime}=x_{1} R \oplus \cdots \oplus x_{n} R \subseteq F$ and $K^{\prime}=K \cap F^{\prime}$. There exists an R-epimorphism $g: K \rightarrow(N: m)$, where $(N: m)=\{r \in R \mid m r \in N\}$, such that $\operatorname{ker} g=K^{\prime}$.

Proof. If $u \in K$, then $u=x_{1} r_{1}+x_{2} r_{2}+\cdots+x_{n} r_{n}+x_{n+1} r_{u}$. Since $0=f(u)=$ $m_{1} r_{1}+\cdots+m_{n} r_{n}+m r_{u}$, it follows that $m r_{u} \in N$, hence that $r_{u} \in(N: m)$. Define $g$ by $g(u)=r_{u}$ for each $u \in K$. Q.E.D.

2. Flat regular quotient rings. We can now state and prove the first main result of this paper: an ideal theoretic characterization of any ring $R$ with $Z\left(R_{R}\right)=$ (0) for which any regular right quotient ring is flat as a left $R$-module. Every ring $R$ that satisfies $Z\left(R_{R}\right)=(0)$ has a regular right quotient ring, namely the MRQ ring [7].

THEOREM 2.1. Let $R$ be a ring with $Z\left(R_{R}\right)=(0)$. If $Q$ is a regular right quotient ring of $R$, then the following statements are equivalent:

(a) $Q$ is flat as a left $R$-module.

(b) Every finitely generated right ideal I of $R$ is EFR.

(c) For any finitely generated right ideal $I$ of $R$ and any element a of $R,(I: a)=$ $\{r \in R \mid$ ar $\in I\}$ is $E F G$.

(d) For any $a \in R,(0: a)=\{r \in R \mid a r=0\}$ is $E F G$ and if $I_{1}, I_{2}$ are finitely generated right ideals of $R$, then $I_{1} \cap I_{2}$ is EFG.

Proof. (a) implies (d). Let $a \in R$ and $a^{*}: R \rightarrow a R$ be the $R$-epimorphism defined by $a^{*}(x)=a x$ for each $x \in R$. The sequence $0 \rightarrow(0: a) \stackrel{i}{\rightarrow} R \stackrel{a^{*}}{\rightarrow} a R \rightarrow 0$ is then exact, where $i$ is the inclusion map. It follows from (a) and [10, Propositon 1, p. 132] that the following sequence is also exact.

$$
0 \longrightarrow(0: a) Q \stackrel{i}{\longrightarrow} Q \longrightarrow a R Q \longrightarrow 0 .
$$

By regularity of $Q$ there exists idempotent $e$ of $Q$ such that $a R Q=a Q=e Q$ [16]. It follows that (1) splits and, hence, there exists idempotent $f$ of $Q$ such that $(0: a) Q=$ $f Q$. Since $(0: a) Q \cong(0: a) \otimes Q$ it follows by Lemma 1.5 that $(0: a)$ is EFG.

To show the second part of (d) observe that $I_{j} Q, j=1,2$, are finitely generated right ideals of $Q$, hence $I_{j} Q=e_{j} Q$ for idempotents $e_{j}, j=1,2$ of $Q$ [16]. From Lemma 1.10 and (a) we have $\left(I_{1} \cap I_{2}\right) Q=I_{1} Q \cap I_{2} Q=e_{1} Q \cap e_{2} Q$. Regularity of $Q$ now gives $\left(I_{1} \cap I_{2}\right) Q=e_{3} Q$ for some idempotent $e_{3}$ of $Q$ and Lemma 1.5 gives that $I_{1} \cap I_{2}$ is EFG. We have (d). 
(d) implies (c). For any finitely generated right ideal $I$ of $R$ and element $a$ of $R$ the sequence $0 \rightarrow(0: a) \stackrel{i}{\rightarrow}(I: a) \stackrel{a^{*}}{\rightarrow} I \cap a R \rightarrow 0$ is exact $\left(a^{*}(x)=a x\right.$ for each $x \in(I: a))$. From (d) we have $(0: a)$ and $I \cap a R$ are EFG so that $(I: a)$ is EFG follows from Lemma 1.4(a). We have (c).

(c) implies (b). Let $I=\sum_{i=1}^{n} a_{i} R$ be a finitely generated right ideal of $R$. We proceed by induction on $n$. For $n=1, I=a_{1} R$ and $I$ is EFR follows from the exact sequence $0 \rightarrow\left(0: a_{1}\right) \rightarrow R \stackrel{a_{\mathrm{i}}}{\rightarrow} a_{1} R \rightarrow 0$ where $\left(0: a_{1}\right)$ is EFG by (c). Assume now that $A$ is EFR whenever $A$ is a right ideal of $R$ generated by $k$ elements where $k<n<\infty$ and $n>1$. Let $F=x_{1} R \oplus \cdots \oplus x_{n} R$, a free right $R$-module, $F^{\prime}=x_{1} R \oplus \cdots$ $\oplus x_{n-1} R \subseteq F$ and $f: F \rightarrow I$ the $R$-epimorphism defined by $f\left(x_{i}\right)=a_{i}, 1 \leqq i \leqq n$. Let $K=\operatorname{ker} f, I^{\prime}=a_{1} R+\cdots+a_{n-1} R$ and $\bar{f}$ the restriction of $f$ to $F^{\prime}$. The sequences

$$
0 \longrightarrow K \stackrel{i}{\longrightarrow} F \stackrel{f}{\longrightarrow} I \longrightarrow 0
$$

and

$$
0 \longrightarrow K^{\prime} \stackrel{i^{\prime}}{\longrightarrow} F^{\prime} \stackrel{\bar{f}}{\longrightarrow} I^{\prime} \longrightarrow 0
$$

are exact where $i$ and $i^{\prime}$ are inclusion maps. The following exact sequence is obtained from Lemma 1.11:

$$
0 \longrightarrow K^{\prime} \longrightarrow K \stackrel{g}{\longrightarrow}\left(I^{\prime}: a_{n}\right) \longrightarrow 0 .
$$

Now $K^{\prime}$ is EFG in (3) follows from induction assumption and the remark following Definition 1.3. In (4) $\left(I^{\prime}: a_{n}\right)$ is EFG by (c) so $K$ is EFG follows from Lemma 1.4(a). Now (2) gives that $I$ is EFR. We have (b).

(b) implies (a). If $I$ is any finitely generated right ideal of $R(\mathrm{~b})$ and Proposition 1.2 give that $I \otimes Q$ is projective as a right $Q$-module, hence $Z(I \otimes Q)=(0)$. Now (a) follows from Theorem 1.9.

The proof of the theorem is now complete.

REMARKS. It is easy to check that statements (c) and (d) are respectively equivalent to the following statements:

(c') If $I$ is any right ideal of $R$ which is EFG and $a$ is any element of $R$, then $(I: a)$ is $\mathrm{EFG}$.

(d') $(0: a)$ is EFG for any element $a$ of $R$. If $I_{1}$ and $I_{2}$ are EFG right ideals of $R$, then $I_{1} \cap I_{2}$ is EFG.

Thus in statements (c) and (d) of the preceding theorem, "finitely generated" can be replaced by EFG.

The following are examples of rings belonging to the class of rings of Theorem 2.1:

(i) A right Noetherian ring $R$ satisfies statement (d), for example, and if $Z\left(R_{R}\right)=$ (0) then its MRQ ring is regular, hence flat as a left $R$-module.

(ii) If $R$ is right semihereditary then $Z\left(R_{R}\right)=(0)[5$, p. 426] and any finitely generated right ideal of $R$ is EFR since it is projective. 
(iii) If $R$ is finite-dimensional as a right $R$-module [13] and if $Z\left(R_{R}\right)=(0)$, then any right ideal of $R$ is EFG [13, Theorem 1.3] so (c), for example, holds. Such rings are characterized in [13] and are those which have a semisimple (with d.c.c.) MRQ ring $Q$. Thus the semisimple MRQ ring $Q$ of a ring $R$ is flat as a left $R$ module [13, Theorem 2.7, p. 119].

Finally, we remark that statements (b), (c) and (d) of the theorem are internal characterizations so that if $R$ with $Z\left(R_{R}\right)=(0)$ has a regular right quotient ring, which is flat as a left $R$-module, then every regular right quotient ring of $R$, in particular the MRQ ring of $R$, is flat as a left $R$-module.

We next give a module-theoretic characterization of the rings of Theorem 2.1.

THEOREM 2.2. Let $R$ be a ring with $Z\left(R_{R}\right)=(0)$. If $Q$ is a regular right quotient ring of $R$, the following statements are equivalent:

(a) ${ }_{R} Q$ is flat.

(b) If $M_{R}$ is a finitely generated submodule of $F_{R}$, free module of finite rank, then $M_{R}$ is EFR.

(c) If $M_{R}$ is a finitely generated submodule of $F_{R}$, free of finite rank, and $x \in F$, then $(M: x)=\{r \in R \mid x r \in M\}$ is $E F G$.

(d) Let $F_{R}$ be a free module of finite rank. For any $x \in F,(0: x)=\{r \in R \mid x r=0\}$ is $E F G$, If $M_{1}$ and $M_{2}$ are finitely generated submodules of $F_{R}$, then $M_{1} \cap M_{2}$ is EFG.

Proof. (a) implies (d). Let $F_{R}$ be free of rank $n$ and $x=\left(a_{1}, \ldots, a_{n}\right) \in F$, $a_{i} \in R$. Since $(0: x)=\bigcap_{i=1}^{n}\left(0: a_{i}\right)$, it follows from Theorem $2.1\left(\mathrm{~d}^{\prime}\right)$ that $(0: x)$ is EFG.

To show the second part of (d) we see that (a) implies that the sequences $0 \rightarrow M_{i} \otimes Q \rightarrow F \otimes Q, i=1,2$, and $0 \rightarrow\left(M_{1}+M_{2}\right) \otimes Q \rightarrow F \otimes Q$ are exact. By Lemma 1.6 , the right $Q$-modules $M_{i} \otimes Q, i=1,2$ and $\left(M_{1}+M_{2}\right) \otimes Q$ are $Q$ projective. A further application of Lemma 1.6 gives that the exact sequence $0 \rightarrow M_{2} \otimes Q \rightarrow\left(M_{1}+M_{2}\right) \otimes Q \rightarrow M_{1}+M_{2} / M_{2} \otimes Q \rightarrow 0$ splits. It follows that $M_{1} / M_{1} \cap M_{2} \otimes Q$ is projective as a right $Q$-module since $M_{1} / M_{1} \cap M_{2} \otimes Q \cong$ $M_{1}+M_{2} / M_{2} \otimes Q$. Now the exact sequence $0 \rightarrow\left(M_{1} \cap M_{2}\right) \otimes Q \rightarrow M_{1} \otimes Q \rightarrow$ $M_{1} / M_{1} \cap M_{2} \otimes Q \rightarrow 0$ splits and $\left(M_{1} \cap M_{2}\right) \otimes Q$ is finitely generated as a right $Q$-module since $M_{1} \otimes Q$ is. $M_{1} \cap M_{2}$ is EFG, follows from Lemma 1.5, as $Z\left(M_{1} \cap M_{2}\right)=(0)$. We have (d).

(d) implies (c). For any $x \in F$ define $x^{*}:(M: x) \rightarrow M \cap x R$ by $x^{*}(r)=x r$ for each $r \in(M: x)$, clearly an $R$-epimorphism. We obtain the exact sequence $0 \rightarrow(0: x) \stackrel{i}{\rightarrow}(M: x) \stackrel{x^{*}}{\rightarrow} M \cap x R \rightarrow 0$ and (c) follows from Lemma 1.4(a), since $(0: x)$ and $M \cap x R$ are both EFG by (d) and $Z((M: x))=(0)$ as $(M: x) \subseteq R_{R}$.

(c) implies (b). Let $M_{R}=\sum_{i=1}^{n} m_{i} R$ be a finitely generated submodule of $F_{R}$. We proceed by induction on $n$, the number of generators of $M$. For $n=1$ we have $M_{R}=m_{1} R \subseteq F$ and $\left(0: m_{1}\right)$ is EFG by (c). Now $M_{R}$ is EFR follows from the exact 
sequence $0 \rightarrow\left(0: m_{1}\right) \stackrel{i}{\rightarrow} R \stackrel{m_{1}^{*}}{\rightarrow} m_{1} R \rightarrow 0$ where $m_{1}^{*}(r)=m_{1} r$ for each $r \in R$. Let $n>1$ and assume that $L_{R}$ is EFR whenever $L_{R} \subseteq F_{R}$ and $L_{R}$ is generated by $k$ elements, $k<n<\infty$. The argument used in showing "(c) implies (b)" for Theorem 2.1 can be applied here to show that $M_{R}=\sum_{i=1}^{n} m_{i} R$ is EFR.

(b) implies (a). Statement (b) of Theorem 2.1 is a special case of (b) of Theorem 2.2, so we have (a).

This completes the proof of the theorem.

We close with a word about a class of commutative nonsingular rings $R$ for which no regular quotient ring is $R$-flat.

If $R$ is a commutative ring and $M$ a maximal ideal of $R$, we denote by $R_{M}$ the quotient ring of $R$ with respect to the multiplicatively closed set $R-M[2$, p. 141] (and [4]).

Let $\left\{R_{\alpha}: \alpha \in A\right\}$ be a collection of valuation domains not all fields and all containing a common field $K$. It follows from a result of Nagata [12] that there exists a ring $T$ with the following properties:

(a) For each $\alpha \in A$ there exists a maximal ideal $M_{\alpha}$ of $T$ such that $T_{M_{\alpha}} \cong R_{\alpha}$.

(b) For every maximal ideal $M$ of $T, T_{M} \cong K$ or $T_{M} \cong R_{\alpha}$ for some $\alpha \in A$.

(c) $T$ is its own total quotient ring [4, p. 115].

From these we further deduce the following properties of $T$ :

(1) $T$ is not regular [4, Theorem 1, p. 110].

(2) $T$ is not semihereditary [4, Theorem 2, p. 113].

(3) If GWD (T) denotes the global weak dimension of $T$, then GWD $(T) \leqq 1$ [4, Proposition 11, p. 116].

It follows from (3) and [5, p. 426] that $Z(T)=(0)$. Now any commutative ring $T$ with GWD $(T) \leqq 1$ and maximal quotient ring $T$-flat, is semihereditary [14, Theorem 2.10]. From this and (2) it follows that the maximal quotient ring of $T$ is not $T$-flat. From a remark following Theorem 2.1, no regular quotient ring of $T$ is $T$-flat.

\section{REFERENCES}

1. N. Bourbaki, Algèbre commutative, Chapters 1-2, Hermann, Paris 1961.

2. H. Cartan and S. Eilenberg, Homological algebra, Princeton Univ. Press, Princeton, N. J., 1956.

3. S. U. Chase, Direct products of modules, Trans. Amer. Math. Soc. 97 (1960), 457-473.

4. S. Endo, On semi-hereditary rings, J. Math. Soc. Japan 13 (1961), 109-118.

5. E. R. Gentile, Singular submodule and injective hull, Indag. Math. 24 (1962), 426-433.

6. C. U. Jensen, A remark on arithmetical rings, Proc. Amer. Math. Soc. 15 (1964), 951-954.

7. R. E. Johnson, The extended centralizer of a ring over a module, Proc. Amer. Math. Soc. 2 (1951), 891-895.

8. R. E. Johnson and E. T. Wong, Self-injective rings, Canad. Math. Bull. 2 (1959), 167-173.

9. I. Kaplansky, Projective modules, Ann. of Math. 68 (1958), 372-377.

10. J. Lambek, Lectures on rings and modules, Blaisdell, Waltham, Mass., 1966.

11. L. Levy, Torsion-free and divisible modules over non-integral domains, Canad. J. Math. 15 (1963), 132-151. 
12. M. Nagata, Some remarks on prime divisors, Mem. Coll. Sci. Univ. Kyoto. Ser. A. Math. 33 (1960), 297-299.

13. F. L. Sandomierski, Semisimple maximal quotient rings, Trans. Amer. Math. Soc. 128 (1967), 112-120.

14. -, Nonsingular rings, Proc. Amer. Math. Soc. 19 (1968), 225-230.

15. R. G. Swan, Groups with periodic cohomology, Bull. Amer. Math. Soc. 65 (1959), 368-370.

16. J. von Neumann, On regular rings, Proc. Nat. Acad. Sci. U. S. A. 22 (1936), 707-713.

\section{UNIVERSITY OF WISCONSIN,}

MADISON, WisCoNSIN 